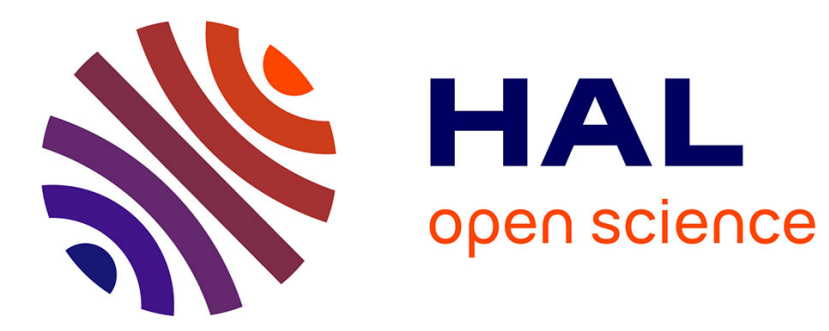

\title{
L'entremise du récit du chercheur : une manière d'aborder le rôle des discours et des médias dans la patrimonialisation
}

Cécile Tardy

\section{- To cite this version:}

Cécile Tardy. L'entremise du récit du chercheur: une manière d'aborder le rôle des discours et des médias dans la patrimonialisation. Culture et Musées, 2003, 1 (1), pp.109-135. 10.3406/pumus.2003.1169 . hal-03279343

\section{HAL Id: hal-03279343 https://hal.science/hal-03279343}

Submitted on 6 Jul 2021

HAL is a multi-disciplinary open access archive for the deposit and dissemination of scientific research documents, whether they are published or not. The documents may come from teaching and research institutions in France or abroad, or from public or private research centers.
L'archive ouverte pluridisciplinaire HAL, est destinée au dépôt et à la diffusion de documents scientifiques de niveau recherche, publiés ou non, émanant des établissements d'enseignement et de recherche français ou étrangers, des laboratoires publics ou privés. 


\section{L'entremise du récit du chercheur : une manière d'aborder le rôle des discours et des médias dans la patrimonialisation}

Cécile Tardy

\section{Citer ce document / Cite this document :}

Tardy Cécile. L'entremise du récit du chercheur : une manière d'aborder le rôle des discours et des médias dans la patrimonialisation. In: Culture \& Musées, $n^{\circ} 1,2003$. Nouveaux regards sur le patrimoine (sous la direction de Jean Davallon) pp. 109-135;

doi : https://doi.org/10.3406/pumus.2003.1169

https://www.persee.fr/doc/pumus_1766-2923_2003_num_1_1_1169

Fichier pdf généré le 18/04/2018 


\title{
Resumen
}

El articulo empieza con el relato de una operación de protección de la naturaleza en un parque natural regional. Vemos que en un primer nivel tiene lugar una prueba de conversion in situ de un espacio rural en patrimonio, cuya originalidad reside en el enfoque comunicacional de este proceso de patrimonialización. En un segundo nivel, este ejemplo sirve de ocasión al autor del articulo para poner en escena su modo de escritura narrativa en tanto estrategia de comprensión de dicho proceso. El centro de todo radica en esto : probar que el relato permite tratar los discursos que circulan entre actores, a propósito de objetos, y mas ampliamente de espaciós, a volverse patrimonios, dando a conocer lo que pasa en la sociedad que los propulsa y los intercambia, situando, por lo tanto, la patximonialización como un proceso comunicacional que tiene una operatividad social y no ùnicamente simbólica.

\section{Résumé}

L'article commence par le récit d'une opération de protection de la nature dans un parc naturel régional. On voit se jouer à un premier niveau une épreuve de conversion in situ d'un espace rural en patrimoine, l'originalité résidant dans l'approche communicationnelle de ce processus de patrimonialisation. À un second niveau, cet exemple est l'occasion pour l'auteur de l'article de mettre en scène son mode d'écriture narrative comme stratégie de compréhension de ce processus. Le cœur du propos est bien là : montrer que le récit permet de traiter les discours qui circulent entre des acteurs, à propos d'objets (et plus largement d'espaces) en " devenir patrimoine », en montrant ce qui se passe dans la société qui les porte et les échange, donc en situant la patrimonialisation comme un processus communicationnel ayant une opérativité sociale et pas seulement symbolique.

\begin{abstract}
The article begins with the tale of a nature preservation project in a regional natural park. On the first level, we witness the on-site struggle to convert a rural spot into a part of the cultural heritage. The originality of this event rests in the communication aspect of this heritage-making process. On a second level, this example gives the article 's author the chance to put her narrative writing style on stage as a strategy to help us understand this process. This is the heart of the matter. The article shows that narrative allows us to deal with those discourses that circulate between acting agents concerned with objects and, more broadly speaking, with spaces that are becoming parts of the cultural heritage. This is done by showing what happens in the society that possesses and exchanges these objects and spaces. Heritage-making is thus contextualised as a communicational process that functions on a social, and not just symbolic, level.
\end{abstract}




\section{L'ENTREMISE DU RÉCIT DU CHERCHEUR : UNE MANIERE D'ABORDER LE RÔLE DES DISCOURS ET DES MÉDIAS DANS LA PATRIMONIALISATION}

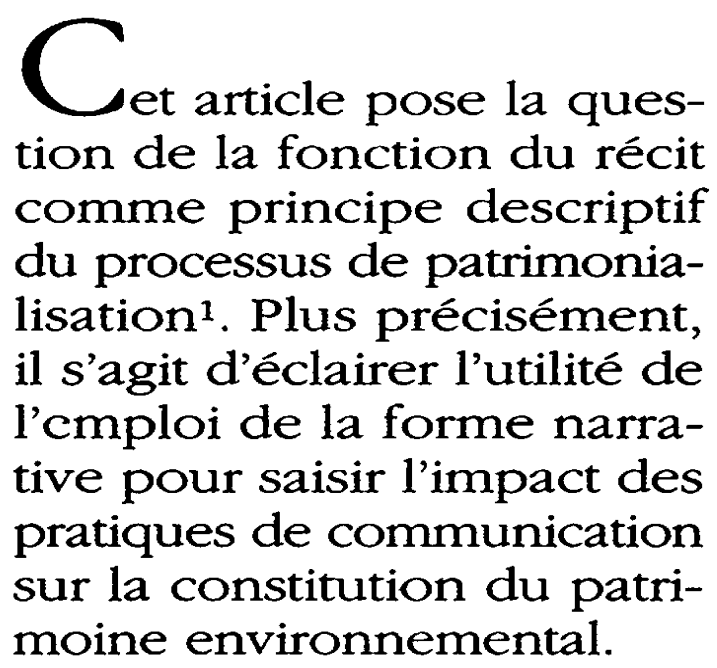

La question est de savoir comment la mise en ouvre du récit par le chercheur permet de rendre compte de l'action sociale de patrimonialisation à partir des discours qui s'échangent entre des acteurs, à propos d'objets, et plus largement d'espaces, en devenir patrimoines. Avant d'aborder cet aspect méthodologique, un temps est consacré à l'application de la méthode narrative sur un cas concret : nous faisons le récit d'une opération de protection de la nature initiée par le parc naturel régional Livradois-Forez.

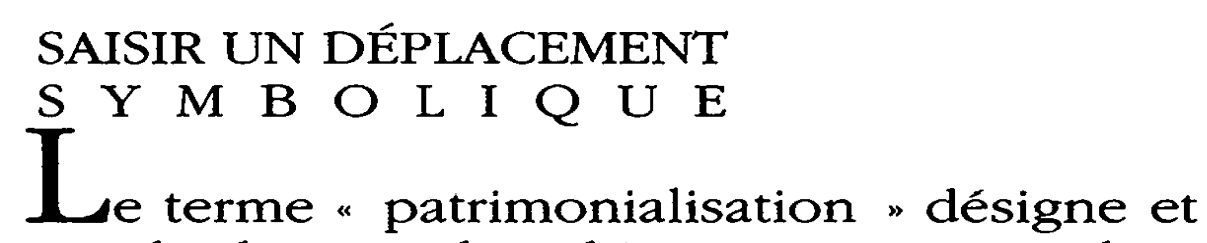
définit un processus de devenir des objets - et non un objet défini - qui implique un phénomène relationnel entre des individus, des groupes et des objets, au cours duquel les uns et les autres se construisent comme acteurs et comme patrimoines. L'exemple fondateur de ce retournement de l'approche du patrimoine est le cas du patrimoine rural dans sa définition élargie, du bâti aux savoir-faire, paysages, espèces animales... Immergé dans l'actualité et la vie quotidienne, le patrimoine rural invite à réintroduire fortement la question des enjeux économiques. Or, il nous intéresse d'observer et d'analyser l'accrochage entre ceux-ci et les enjeux symboliques, entre le vécu matériel de ces patrimoines et leur caractère symbolique. Cette imbrication ne peut se saisir qu'en prenant en compte le 
" devenir patrimoine " de ces choses de ta ruralité, en se situant au plus près de leur processus de fabrication. Ainsi, nous ne pensons pas le patrimoine dans une perspective historique et artistique, ni dans une perspective de légitimation par les sciences pour une entrée au musée ou une utilisation politique, mais nous le posons comme objet problématique en tant qu'objet d'énonciation, objet de sociabilité, de partage : un objet inséré dans des pratiques de communication qui le mettent en scène, le manipulent, l'élaborent, lui donnant un sens patrimonial.

Cette manière de poser le problème de la patrimonialisation s'appuie sur l'expérience d'institutions territoriales : les parcs naturels régionaux, et plus précisément celui du LivradoisForez. Si ces structures disent travailler à un nouveau modèle de développement qui cherche à harmoniser protection de l'environnement et développement économique, nous les considérons pour notre part comme des " laboratoires " où s'élabore une nouvelle manière de concevoir notre rapport à l'espace rural. Or la spécificité de ces territoires ruraux étant de contenir la vie, naturelle et humaine, ce travail ne peut se faire par le déplacement physique d'une matière dans les murs d'un musée ou dans l'enceinte d'un écomusée. Alors, comment muer un espace rural qui vit sans le déplacer physiquement dans un lieu resignifiant ? Nous pensons que l'analyse doit s'orienter sur le rôle des discours dans cette transformation in situ de l'espace rural en signes patrimoniaux. En effet, dans cette conversion, la communication joue un rôle déterminant puisqu'il faut à nouveau désigner, légitimer, argumenter, questionner, réglementer, conceptualiser le rapport à cet espace.

L E R É C I T D E L A PATRIMONIALISATION D U H A U T FORE Z C ommençons par exposer le récit de la constitution de l'espace du Haut Forez comme patrimoine à l'initiative du parc naturel régional Livradois-Fore $z^{3}$, les questions d'ordre méthodologique étant réservées à la partie suivante ${ }^{4}$.

Cette opération de patrimonialisation consiste à délimiter un espace physique pour le fixer en un territoire soumis au régime des sites classés au titre des monuments historiques et des sites (loi de 1930). Ce que nous nous attachons à décrire est la mise en discours de cette action (de nature essentiellement scientifique, institutionnel et médiatique) et ce qu'elle fait advenir pour l'espace du Haut Forez : faire voir, croire, connaître et reconnaître, à la fois un nouveau sens, patrimonial, et la nouvelle collectivité attachée à cet espace, le parc Livradois-Forez. 
Ce projet de protection de la nature se révèle être une opération culturelle visant à réaliser une nouvelle organisation sociale.

Au DÉBut DES ANNÉES 1980 : D'UN PROJET DE CONNAISSANCE À UN PROJET DE PROTECTION DE LA NATURE

À partir des années 1960 , le versant auvergnat des monts du Forez est en plein déclin démographique et économique, avec l'abandon de l'activité principale traditionnelle, l'agropastoralisme. Les années 1970 apparaissent comme des années de transition avec une activité ovine qui tend à se développer en se substituant aux troupeaux bovins. Les années 1980 annoncent un intérêt nouveau pour l'espace montagnard, timide sur le plan agricole mais dynamique sur le plan scientifique dans le domaine environnemental.

Le champ de l'écologie découvre là un site peu exploré et, qui plus est, propice au développement de ce type de recherche par ses richesses naturelles. Les universités stéphanoises et clermontoises alimentent cette connaissance écologique. Un chercheur de l'université auvergnate participe à l'impulsion de ce mouvement avec sa thèse de doctorat (1983) et sa thèse d'État en phytoécologie sur le terrain des Hautes Chaumes. Progressivement se structure une nouvelle perception du site à travers sa valeur écologique exceptionnelle. L'espace est défini, nommé : c'est la partie haute du massif qui focalise le regard des chercheurs écologistes, tout particulièrement les "Hautes Chaumes" ", espace sommital dénudé, et plus globalement le " Haut Forez ", qui comprend les Hautes Chaumes et l'étage forestier limitrophe intéressant pour ses sapinières et hêtraies anciennes, cet ensemble géographique se situant au-dessus de 1200 mètres et de l'habitat permanent ${ }^{6}$.

La création du parc naturel régional Livradois-Forez, officialisée en 1986, va permettre à ce chercheur de passer à une dimension de gestion de la nature. Le parc lui délègue la responsabilité du secteur " environnement " jusqu'en 1991, plaçant l'écologie scientifique dans une situation de gestionnaire des milieux naturels. Cette collectivité territoriale va exposer son point de vue en 1993 dans l'ouvrage intitulé Les Hautes Chaumes du Forez. Diagnostic écologique pour la gestion d'un espace sensible - publié avec le partenariat des géographes de l'université de Saint-Étienne et du parc naturel régional LivradoisForez - et présente l'engagement d'une procédure de protection dès 1987 concernant le classement à l'inventaire des sites de deux vallées glaciaires situées au coeur des Hautes Chaumes et l'inscription à l'inventaire des sites d'un espace périphérique dit de grand intérêt paysager et scientifique.

Dans les années 1990 paraît un livret à l'adresse du grand public qui révèle la face cachée du Haut Forez, c'est-à-dire ses 
composantes naturelles, et participe ainsi à la reconstruction de la représentation des monts du Forez. Le président du parc Livradois-Forez, qui préface le document, érige l'image forte du " monument naturel ", parle des Hautes Chaumes comme une " pièce rare ", cite l'" étage subalpin ", la "flore exceptionnelle ", "le merle à plastron ".

Subjugué par ce domaine patrimonial qui couple les intérêts culturel et naturel, le parc naturel régional Livradois-Forez, fort de sa vocation patrimoniale, s'engage dans la reconstruction de l'espace du Haut Forez avec l'aide des écologistes scientifiques. Un nouveau langage, une nouvelle carte d'identité, viennent signer une nouvelle naissance pour les monts du Forez, le projet de classement et d'inscription correspondant métaphoriquement à l'acte de baptême. Ce geste de sauvegarde sera-t-il accepté par la population?

1988-1993 : L'AFFAire du ClASSEMENT du Haut Forez À TRAVERS SA MISE EN DISCOURS DANS LA PRESSE

Notre enquête de terrain a donné lieu à la réalisation de la revue de presse portant sur l'épisode du classement du Haut Forez", reconstruisant ainsi le "récit médiatique" sur ce sujet ${ }^{8}$. Notre propre récit relit le récit médiatique en montrant comment il participe de la patrimonialisation du Haut Forez à travers ses discours et les médias en jeu et en le rapprochant, dans l'analyse de ce mouvement social, d'autres discours tels ceux présentés précédemment des scientifiques et de l'institution du parc.

L'analyse de ce récit médiatique nous a amenés à articuler notre propre récit en cinq séquences, correspondant aux temps forts de l'épisode étudié, en partant du moment de l'exposition au public de la démarche de protection du site par le parc Livradois-Forez en 1988 à l'obtention de son classement cinq ans plus tard, en 1993.

\section{1) Séquence I : Le moment inaugural}

Le porteur du projet de classement et d'inscription à l'inventaire des sites du Haut Forez central, le parc naturel régional Livradois-Forez, organise successivement dans les trois communes concernées par le périmètre - Job, Valcivières, Vertolaye - trois réunions publiques de sensibilisation et d'information en mars 1988. C'est le moment où le parc LivradoisForez propose à la population de se positionner par rapport à la définition écologique de l'espace du Haut Forez développée précédemment par les recherches scientifiques. Chaque réunion donnera lieu à un article dans la presse quotidienne, La 
Montagne. C'est sur la base du corpus de ces trois articles que l'on suit le déroulement de ces réunions publiques.

Les termes de la formulation

Les titres des articles désignent l'espace dont il est question comme " le Haut Forez ". L'explication du périmètre de la zone à classer utilise des critères comme la limite administrative départementale, l'exclusion de l'habitat permanent, donc des hommes qui vivent sur ce territoire, la protection d'un bien commun, les valeurs environnementales du site dégagées par les études scientifiques (" intérêts ", " richesse ", "spécificité ", " remarquable ", " variée et spécifique ", " fragile "). Le parc LivradoisForez, dans l'exercice de ce moment inaugural en face-à-face avec l'assistance, tente essentiellement de faire reconnaître le savoir environnemental accumulé sur le Haut Forez.

\section{Une réception mitigée}

Avec ces réunions, on assiste à un déplacement de la dimension cognitive de la représentation spatiale, base du projet du parc, sur le terrain des usages. Les questions ne portent pas sur les richesses naturelles du site à protéger, comme si l'écologie scientifique était trop éloignée des préoccupations de l'assistance, mais sur le contenu de la loi, sur ce que l'on peut faire et ne pas faire dans le cadre d'une procédure d'inscription et de classement. L'épreuve du passage de l'énoncé de la procédure de classement et d'inscription du parc à l'assistance a donc contribué à la suppression de la dimension cognitive du projet pour n'en retenir que son application, qui devient le seul terrain de dialogue, l'unique sujet propice à la production d'un échange entre les deux entités présentes. À travers ces remarques, le classement apparaît comme une procédure de déqualification de certaines pratiques au profit d'autres, l'assistance, dite " inquiète ", interprète la notion de bien commun comme une atteinte aux droits privés et interroge donc le porteur du projet sur ses droits en cas de classement du site. De même, l'impact touristique envisagé semble perçu comme une stérilité sociale et économique, comme un processus de muséification.

Le journaliste affiche et rend visible deux mondes qui définissent en s'opposant le Haut Forez : l'écologie et l'économie. Même si la frontière semble franchissable - "Écologie et économie peuvent aller de pair " -, il reste du chemin à faire pour que les uns et les autres acceptent d'échanger, voire de partager leurs valeurs respectives.

Toutefois, sur le plan du paraître, cette première épreuve semble réussie avec l'amorce du projet par la mise en place d'un comité de pilotage lors de la dernière réunion. Ainsi, l'épreuve des réunions publiques aboutit à une qualification minimale du projet du parc Livradois-Forez qui se poursuit 
malgré une base des plus fragiles, faite d'incertitude, de méfiance et d'incompréhension.

2) Séquence II : Le débat médiatique. Lorsque le champ s'organise

Dans cette seconde séquence, on voit se mettre en place une structuration du champ : installation de deux médias qui donnent à lire différentes opinions en même temps qu'ils prennent position par rapport au projet de classement ; désignation par ces journaux, non plus d'une assistance floue, mais d'acteurs porteurs de projets qui tendent à déstabiliser ou au contraire à conforter le projet initial.

Le signal d'alarme

En juin 1989, le journal La Montagne rapporte une tension dans le déroulement du projet de classement du site naturel : "Vallées glaciaires du Haut Forez, des aménagements en perspective " titre l'article. On relève l'opposition citée précédemment entre le savoir écologique et le domaine de l'action : vallées glaciaires $v s$ aménagements. L'image des "vallées glaciaires " renvoie au Haut Forez, précisément au site à protéger, tandis que sous le thème de l'aménagement se profile une future agression de cet espace dit fragile avec deux opérations : vol libre et route goudronnée. Le projet du parc se trouve ébranlé.

\section{La réaction médiatique}

À partir de 1989, un nouveau journal investit la scène médiatique du classement : le journal auvergnat La Galipote - du nom d'un animal légendaire. Ce journal est créé en 1979 par un journaliste, en opposition à la presse d'information quotidienne, dans le but de fournir une autre information permettant au lecteur d'apprendre et de réfléchir, donc d'améliorer sa compréhension du monde, établissant une communication entre individus et non entre pouvoirs institués (La Galipote 1/1979). C'est une presse d'investigation et d'information critique. Désormais, deux journaux rendent compte du déroulement du classement, La Montagne et La Galipote.

La Galipote explore l'espace du classement dès son premier sous-titre : "Le Haut Forez, site classé ? La dégradation galopante des Hautes Chaumes balaie les réticences ". C'est le point de vue des environnementalistes et amoureux de la nature qui est ici revendiqué, le Haut Forez devenant " les Hautes Chaumes ".

La nomination des Hautes Chaumes renvoie à un vaste espace dénudé en altitude, c'est-à-dire le sommet des monts du Forez, étendue de landes composée d'un étage subalpin et d'un étage montagnard. L'installation de l'image de ce plateau d'altitude étiré du nord au sud des monts du Forez, et précisément 
délimité par ses caractéristiques géographiques, instaHe une dimension spatiale horizontale. Le recoupement avec le texte du journal La Montagne rapportant l'exclusion de l'habitat permanent du périmètre à inscrire et classer renforce cette perception horizontale d'un espace coupé de ses versants. Le journal revient sur les connaissances scientifiques accumulées par le parc Livradois-Forez en décrivant l'espace dans les mêmes termes. Cet ensemble patrimonial naturel et culturel revêt des valeurs d'" originalité ", d'" authenticité " et de " pittoresque ".

La Galipote structure la défense du projet de classement en recensant et en désignant les projets alliés ou opposés à celui du classement. Pour mobiliser ces voix du terrain, La Galipote procède par apport de documents écrits ou oraux. Du premier type de documentation relève des avis écrits parvenus en souspréfecture et un dossier épais sur le projet de vol libre. Le deuxième type de documcntation compte une série d'interviews réalisées par La Galipote, citées et commentées dans le texte journalistique ou présentées sous la forme de sous-textes indépendants intitulés "les échos de la vallée ".

Le projet le moins développé, car à l'arrêt depuis son lancement en 1987, est celui de la réintroduction du grand tétras par la fédération des chasseurs dans les vallées proposées au classement. Le coq de bruyère aurait pu être un allié idéal du projet de classement dans la mesure où sa survie est conditionnée à la protection du milieu naturel où il est réintroduit.

Deux acteurs associatifs apportent leur soutien au classement : pour le Conservatoire régional de l'habitat et des paysages (CRHP9), qui va devenir un des alliés principaux du parc Livradois-Forez, le projet de classement devrait permettre de stopper la banalisation du site par le tracé de pistes et la menace d'une future " route touristique des crêtes ". Pour l'Association de protection et de promotion des produits naturels de Peyre-Mayou (APPN) qui organise tous les 15 août sur ce site des Hautes Chaumes la "Fête de la myrtille ", le classement est conçu comme une labellisation de l'espace et cles produits qui en sont issus ainsi qu'une protection contre les dégâts causés par les véhicules $4 \times 4$ et les pilleurs de myrtilles.

Au début du lancement de la procédure du classement, les propriétaires de la vallée du Fossat (inscrite dans le projet de classement), mécontents du développement précédent du domaine nordique pour le ski de fond, trouvent que le classement est l'occasion de penser globalement les projets.

Un autre allié du projet de classement est " l'article 1910 ", tel que l'explique le berger du troupeau ovin de la coopérative d'estive des Nerses. La très longue interview qu'il donne au journal le situe comme un porte-parole essentiel dans la procédure de classement. Selon lui, le classement serait un pas vers la reconnaissance du Haut Forez comme zone sensible du 
point de vue de l'environnement ce qui la rendrait éligible pour bénéficier des subventions européennes. Si le berger est sans contexte un allié du projet de classement, les membres de la coopérative ne sont pas convaincus. Par conséquent, leurs terrains se situant à cheval sur le site à classer et sur le terrain du futur projet de parc de vol libre (conflit à venir), ils peuvent être un partenaire stratégique ou un opposant de poids.

L'opposition la plus forte commence à se structurer autour de deux projets, situés au coeur du site proposé au classement, qui vont s'avérer complémentaires : celui d'aménagement de voirie et celui du développement de l'activité libériste. Les deux porteurs de projet vont très vide devenir les personnages forts des discussions et conflits à venir : il s'agit du maire de la commune de Job (l'une des deux communes dont les terrains sont directement concernés par le projet de classement et celui de vol libre), et par le président du Delta Club Livradois-Forez. Ce dernier insiste sur la dimension verticale du massif : ce n'est plus la hauteur qui est pertinente mais la " dénivelée ", donc la différence d'altitude entre deux extrémités, le haut et le bas. Alors que cet opposant valorise le domaine aérien, arguant, à l'image du maire de Job, qu'il ne voit pas "en quoi des gens qui, en delta-plane, volent à 200 ou 300 mètres de hauteur menaceraient le site ", les pro-classements scrutent les impacts terrestres du vol libre. La Galipote pointe tous les aménagements du site nécessités par le vol libre.

En réalisant ce tour d'horizon, La Galipote recense les avis de certaines administrations et d'élus adressés en sous-préfecture en août, septembre, novembre 1988 et janvier 1989, face au projet de protection proposé par le parc. On note que les élus locaux refusent en général de prendre une position ouverte, et les élus des deux communes (Job et Valcivières) concernées directement par le projet de classement penchent plutôt vers l'opposition.

La polarisation vol libre / classement

L'annonce par le journal La Montagne du déroulement des Internationaux de France de vol libre sur le site en projet de classement durant l'été 1990 précipite le conflit ainsi que le positionnement des deux médias journalistiques. La presse locale La Montagne s'affiche de plus en plus partisane dans sa façon de traduire le discours de façade de $M$. T., porteur du projet de vol libre sans jamais le questionner, plaçant le parc dans la situation de gêneur et le projet de classement comme une contrainte pour le développement économique et touristique de la région.

Selon La Galipote, " une erreur de parcours issue de quelques articles trop avancés dans le journal La Montagne mettait le feu aux poudres ". Ce journal d'investigation critique pointe les 
faiblesses des projets de vol libre et de voirie et responsabilise M. T., ainsi que le conseil municipal de Job, le conseil régional et le SIVOM d'Ambert. La Galipote lance un appel à la vigilance : la coopérative d'estive des Nerses met en vente des terrains inutilisables sur le plan agricole, mais stratégiques dans le projet de classement, en arguant de la nécessité d'une desserte agricole et voyant dans les partisans du vol libre de futurs acquéreurs.

Le point crucial est le moment de l'entrecroisement des forces aux rochers de la Pauze convoités à la fois comme aire de décollage par $\mathbf{M}$. T. et la fédération de vol libre et comme des richesses naturelles à protéger par le conservatoire. Le résultat sera l'achat par le Conservatoire régional de l'habitat et du patrimoine naturel (CRHPN ${ }^{11}$ ) en 1990 d'une parcelle de 22 hectares située sous le promontoire des rochers de la Pauze. L'arrivée du CRHPN est capitale pour le site et le projet de classement car l'association va pallier l'impuissance du parc LivradoisForez, coincé dans des débats politiques qui n'en finissent pas, en apportant ses moyens d'action : l'achat de terrain et donc le contrôle du foncier. Cette association, informe le journal critique, agit " grâce au soutien financier du WWF France, mais surtout grâce à une souscription ". Son action est d'ordre public : le CRHPN " souhaite associer les gens à la gestion du patrimoine ". Il s'agit d'" engager un travail de fond et de sensibilisation à la protection de l'environnement, d'utiliser le site pour initier à la découverte de l'environnement ".

Le CRHPN fait entendre sa voix par l'intermédiaire du journal La Galipote où écrit son principal militant pour le Haut Forez. Il est l'auteur de l'encart signalant l'achat des rochers de la Pauze et d'un important article dans lequel il relate une dernière tentative : le camp des pro-classement dépose une demande d'instance de classement auprès du ministère de l'environnement, sous le nom de la Fédération départementale pour l'environnement et la nature du Puy-de-Dôme (FDEN 63) au printemps 1990. La Montagne rappelle que l'accord de l'instance bloquerait tous travaux sur le site pendant douze mois, ce qui équivaudrait à paralyser le projet de vol libre, et surtout les internationaux fixés pour la fin de l'été 1990. La Galipote met l'accent sur les forces en présence du côté des partisans en citant les alliés de l'opération. Cette crise donne le signal d'une nécessaire négociation afin de calmer les esprits. Deux réunions plus tard, en présence de la sous-préfecture, du président du parc et du président de la ligue d'Auvergne de vol libre, un accord se dessine : le déclassement de cinq parcelles concernées par le projet d'instance sur les rochers de la Pauze permettrait de réaliser un morceau de voirie. Ce qui n'est que " compromis " pour La Montagne devient une " soumission "sous le regard de La Galipote.

La compétition de vol libre se déroulera donc durant l'été 1990. Un coup est porté au projet de classement, mais les 
alliés du classement sont désormais propriétaires fonciers sur le site des rochers de la Pauze. "Un petit coin de parapluie " chantonne La Galipote à son propos.

\section{3) Séquence III : L'enclenchement de la démarche}

Les débats houleux autour du vol libre s'étant calmés, le parc Livradois-Forez installe à nouveau son projet de classement dans l'actualité. C'est la direction régionale à l'Architecture et à l'Environnement (DRAE) qui procède à l'opération.

Au printemps 1991, la DRAE lance " l'enquête préalable au projet de classement du site du Haut Forez " destinée à recueillir l'avis de la population et des élus. Un avis favorable permettrait de mettre en marche le processus de classement. La Montagne insiste sur cette relation entre l'enquête et le classcment, montrant l'importance d'une participation active de la population et lui donnant les informations nécessaires pour sa participation.

En exacerbant l'opposition parc vs commune de Job, en limitant dans son sous-titre le porteur du projet au seul parc et à la protection unique du patrimoine naturel - " Pour le parc Livradois-Forez, l'objectif est de protéger le patrimoine naturel " -, en mettant en valeur, dans la légende de la carte de situation, la surface comme détail alarmiste - " 700 hectares sont concernés "-, avec d'autre part l'énoncé un rien moqueur du probablement " naturel "à propos de la lisière forestière supérieure, en franchissant le pas de la contrainte vers l'" interdiction ", la presse quotidienne La Montagne n'incite pas la population à cautionner le projet. En écrivant : " Un problème qui renvoie au débat de fond : la conciliation entre la protection de l'environnement et le développement économique ", le journaliste tend à limiter le choix de son lecteur entre " pour l'environnement " ou " pour le développement ". Le débat de fond annoncé s'avère être un non-débat.

La Galipote donne les résultats en faisant le décompte des voix favorables ou non ou encore les avis non exprimés. Il ressort que les tensions tendent à se déplacer de la commune de Job - qui dit oui sous certaines réserves comme l'" ouverture de pistes dans la montagne "- vers celle de Valcivières. Sous le " non " de Valcivières se profile la population des élus chasseurs et des propriétaires. Mais quelle importance? "Un verdict sans appel "souligne La Galipote. Une autre presse, la revue de la Fédération des parcs naturels régionaux (Parcs, $n^{\circ} 14 / 1991$ ) fait paraître l'avis favorable d'un agriculteur installé sur la commune de Job depuis 1975. Il est également membre de l'Association de protection et de promotion des produits naturels de Peyre-Mayou (APPN citée précédemment) qui a soutenu le projet de protection du site. 
Mais la suite des événements révèle que les résultats de l'enquête contenaient un silence, celui des personnes opposées au classement, sous-entendu dans le " non " de la commune de Valcivières, et qui vont à présent s'exprimer.

\section{4) Séquence IV: Les opposants d'après-coup}

Des propriétaires se mobilisent

La longue durée du conflit classement / vol libre a laissé le temps à une partie de la population déjà réticente au projet présenté par le parc Livradois-Forez d'accumuler ses rancoeurs, d'autant plus que ses premières appréhensions sont du coup restées sans réponse. N'ayant pas su ou pas voulu s'exprimer lors de l'enquête préalable, certains mécontents choisissent un mode de communication qui leur est propre pour donner leur avis. Aux deux principaux journaux, La Montagne et La Galipote, s'ajoutent de manière plus brève et ponctuelle la lettre locale intitulée L'Éveil de la Dore, et Le Semeur Hebdo qui font paraître des courriers adressés par la population locale de 1991 à 1993. Le collectif des propriétaires de Valcivières exprime ses revendications au sein d'un tract diffusé à l'occasion d'une manifestation organisée en janvier 1992.

Les propriétaires qui s'expriment contre le classement découpent l'espace dévolu au patrimoine commun national en parcelles privées. Ils placent ainsi en surimpression de la dimension spatiale horizontale explorée par les partisans du classement leur propre champ de perception spatiale : la dimension parcellisée de l'espace du Haut Forez. Les personnes qui s'expriment dans ces courriers et ce tract dressent une série d'oppositions telles que " propriétaire natif " / " technocrates de passage "; " planter, couper, nettoyer, débarder " / " des activités mal définies "; elles parlent de " muséification ", de " désert social et économique ", elles opposent " notre montagne " à "vos Hautes Chaumes ". Le parc Livradois-Forez est le premier visé par cette nouvelle vague de mécontentement.

Ce débat a des conséquences sur le site du projet de classement : face aux interdictions de planter des épicéas et de couper les bois - qui semblent selon eux se profiler -, quelques propriétaires vont s'empresser de planter le fond des vallées glaciaires du Fossat et des Reblats en résineux avant la concrétisation du classement annoncé comme éminent par le journal La Montagne. Le conflit le plus spectaculaire se déclenche à l'occasion de la plantation en épicéas de la vallée du Fossat. Le propriétaire refusera l'achat de sa parcelle par la sous-préfecture. La Galipote raconte les épisodes de cette négociation et son impossibilité pour aboutir ; La Montagne relate la victoire du propriétaire dont l'action " contrecarre "le projet de classement. 
Les tentatives de négociation

Il s'agit de trouver un terrain d'entente commun aux différentes parties en présence. Les différentes tentatives de négociation engagées - représentation des propriétaires dans le comité de gestion; des pistes de ski de fond ouvertes comme pistes forestières; subventions dans le domaine agricole - ne transforment pas radicalement la situation.

Ainsi, la municipalité de Valcivières annonce sa démission si le projet de classement aboutit et la réunion du comité de gestion programmée à Valcivières en janvier 1992 devient le lieu d'une manifestation organisée par les propriétaires et les chasseurs. Or les défenseurs du projet de classement refusent cet échange houleux en annulant la réunion de travail et la démarche de concertation.

Au scepticisme de La Galipote sur le parti pris des porteurs du projet de classement répond l'encouragement de la presse locale La Montagne à l'attitude de rupture prise par la commune de Valcivières en la formulant sur le registre héroïque. Le projet de classement semble se trouver dans une impasse.

L'appel à la sauvegarde de la nature et des paysages

Le Conservatoire régional de l'habitat et du patrimoine naturel (CRHPN) achète successivement des parcelles situées au lieu-dit les rochers de la Pauze grâce aux aides du World Wide Fund for Nature (WWF) et du Fonds européen d'orientation et de garantie agricole (FEOGA). Mais c'est grâce à une souscription publique étalée sur 1992 et 1993 que l'acquisition sera définitive. Les partisans du classement se mobilisent en faveur de cet appel à la sauvegarde d'un site qui répond au souhait de protection des milieux naturels, d'opposition au vol libre et à l'exploitation "violente " de la forêt.

Seul le journal La Galipote informe de la stratégie d'acquisition foncière du conservatoire. Elle satisfait ainsi ses lecteurs dont certains sont devenus des participants de la gestion du Haut Forcz par le biais de la souscription publique.

Cette association met en œuvre une stratégie de gestion intéressante. Par son mode d'action, la maîtrise foncière des milieux naturels foréziens, le conservatoire détourne le problème d'opposition entre les propriétaires gestionnaires et les protecteurs de la nature en devenant lui-même propriétaire. Par son statut associatif et son appel à une souscription publique, le CRHPN tend à gommer la ligne de partage entre patrimoine commun et patrimoine privé. L'accès à la propriété va imposer à cet organisme un décentrage de son univers cognitif vers le domaine de la gestion. 
En 1993, le Haut Forez est inscrit sur la liste des sites classés de France. La Galipote et La Montagne commentent cette demi-victoire, de par le rejet du site inscrit à l'inventaire des monuments historiques et des sites (zone périphérique au site classé), de par le détournement des contraintes pour la gestion forestière. Au bilan, il est possible de dire que l'obtention du classement constitue moins une proposition concrète d'une nouvelle gestion du site qu'une victoire symbolique, mais celle-ci représente un bouleversement non négligeable que l'émergence d'un nouveau type de discours, la signalétique sur le site, vient entériner - l'emblème du parc naturel régional est suivi de : "Vous entrez dans un site naturel fragile. "Ces inscriptions in situ n'ont pas une simple fonction informationnelle par rapport au site protégé. Ellcs mettent en jeu une opération sociale et symbolique en constituant son sens désormais patrimonial, en initiant un nouveau mode d'emploi de ce bout de territoire, en initiant également la population locale, les usagers, les visiteurs, à de nouveaux collectifs dont le plus visible est le parc Livradois-Forez, mais également l'association du CRHPN, qui s'inscrit désormais comme propriétaire représentant d'un collectif d'amateurs de la nature - les personnes qui souscrivent aux achats fonciers adhèrent à l'association, participent aux activités proposées -, avec ses projets, ses connaissances, sa manière de voir et de faire, dans la gestion foncière de la montagne.

Il s'agit à présent, après la mise en ouvre d'une description qui articule un ensemble de données discursives recueillies dans le cadre d'un exemple de patrimonialisation de l'environnement, de se situer sur un second plan, d'ordre méthodologique, afin d'expliciter l'utilisation de la méthode narrative pour se saisir de ce processus social et symbolique.

\section{LE CLASSEMENT DES}

D $\quad I \quad S \quad C \quad O \quad U S R \quad S$

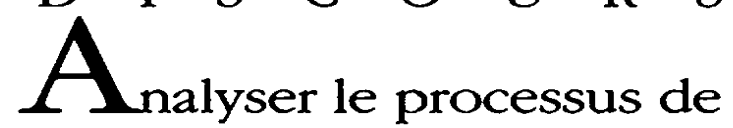

à travers le rôle des discours ouvre sur la question du statut des discours recueillis et donc de leur mode de classement. Le corpus rassemblé pour élaborer le récit du classement du Haut Forez comprend des écrits d'acteurs scientifiques et institutionnels (ouvrages édités, travaux d'études), et une importante revue de presse constituée majoritairement d'articles parus dans deux journaux (un journal quotidien local et un journal d'investigation critique régionale). Dans le cadre d'autres récits, nous avons eu l'occasion de traiter des documents écrits par 
les acteurs eux-mêmes et ayant une importance pour comprendre l'action analysée (statuts associatifs, discours inauguraux, dossier de demande de subvention...) et des témoignages radiophoniques. À ce corpus s'ajoutent les entretiens oraux que nous avons réalisés.

Nous proposons de distinguer au sein des sources à partir desquelles le chercheur peut analyser la patrimonialisation, les discours qui circulent dans la société et que le chercheur extrait de leur vécu pour les ramener au statut de documents de sa recherche, et les discours qu'il provoque par son dispositif d'enquête ${ }^{12}$. En effectuant ce travail de classement des discours sur la base de leurs procédures de construction, il les prépare à une lecture de leur rôle dans la réalité du processus étudié, la patrimonialisation.

Une double opération est réalisée : d'une part des documents contemporains sont transformés en sources, c'est-à-dire en matériaux de base de la recherche et nous renseignent sur le processus de patrimonialisation ; d'autre part, ces sources participent à la construction de la patrimonialisation et sont abordées de ce fait comme des documents performatifs.

Ces matériaux discursifs participent en effet à l'action à deux niveaux : d'une part, ils sont l'instrument par lequel les acteurs médiatisent et se représentent leur action et, d'autre part, ils sont des opérateurs de l'action lorsqu'ils exercent un pouvoir de mobilisation des acteurs et d'inscription de la valeur patrimoniale dans des objets ou des espaces, qui deviennent des véhicules de sens dans la société. Nous avons donc affaire à des discours sur l'action et à des discours dans l'action. L'ensemble du corpus représente la matérialisation de ce sens dans la société et un support de travail et d'analyse de la patrimonialisation d'un point de vue social et sémiotique.

LES DisCOURS MÉdIATIQUES À L'GEUVRE DANS LE PARC NATUREL RÉGIONAL

Certains discours permettent à la patrimonialisation d'être un mouvement durable parce qu'ils ont la capacité de faire durer la valeur patrimoniale indépendamment de la situation d'énonciation et d'échange entre des acteurs. Nous nous intéresserons ici à la presse, matériau le plus mobilisé dans le récit exposé13. À un premier niveau elle réalise une mise en discours de ce qui se passe sur le territoire du parc naturel régional. À un second niveau la presse est à concevoir dans la place qu'elle occupe dans l'action réelle, en prêtant attention à l'usage qui est fait des discours médiatiques par les acteurs et aux représentations patrimoniales des objets comme des acteurs qu'elle contribue à légitimer.

La lecture analytique de la revue de presse effectuée dans le cadre du récit sur le classement du Haut Forez éclate peu à 
peu en direction du contexte social où évoluent les journaux. Le dire est ainsi replacé dans le " cadre des activités organisées où il prend normalement place "(Sharrock et Watson, 1990, p. 23814). On mesure alors les différences de distanciation établies entre chaque type de journal et ses lecteurs. Le rapprochement va jusqu'à brouiller la séparation habituellement établie entre l'univers de production et celui de réception avec l'exemple des acteurs, économiques et associatifs, qui invitent la presse d'opinion à relater leur position ou à le faire euxmêmes par l'écriture d'articles. C'est, d'une part, la position d'auteur qui est objet de changement avec le passage de journalistes professionnels à des acteurs, à des militants d'une action, et d'autre part, la position du lecteur qui tend à devenir participant lorsque la presse lance un appel à participation à l'achat de terrain, ou le stimule pour participer à l'enquête publique - qui prélude la concrétisation ou non du classement de site. L'entrée en jeu de la presse d'investigation représente également l'arrivée d'un nouveau type de public - ses lecteurs " adhérents ", à la fois au journal et à ses représentations sur le rapport de la société à son espace -, plus participatif et militant, qui, bien qu'éloigné de l'espace géographique objet d'enjeux, pourra jouer un rôle non négligeable dans le soutien au processus de classement.

DÉTOURNER LA SÉMIOTIQUE NARRATTVE

Cette nouvelle approche du patrimoine projette la question centrale : comment la mise en récit par le chercheur des données recueillies permet-elle de rendre compte de l'action de patrimonialisation à partir des discours produits ?

La question du récit a mobilisé des chercheurs de différentes disciplines tels qu'historien, anthropologue ou ethnographe, sociologue et linguiste. Leur propos nous interroge quant à la manière dont la mise en récit peut reconstituer et modéliser l'action de patrimonialisation.

Le travail du sémioticien A. J. Greimas, qui observe à partir des années 1960 le récit sous l'angle de sa fabrication, en privilégiant, non pas l'expression, mais le contenu de l'histoire racontée (actions, rôles des personnages, relations entre les actants, etc.) (Gaudreault et Jost, 1990, p. 9-15), nous permet d'aborder le geste technique de la mise en récit.

La sémiotique propose des outils d'analyse qui permettent de s'interroger sur le sens de l'action humaine... à partir des discours. Un problème d'ordre épistémologique se pose concernant leur utilisation car il n'est nullement question de réduire la patrimonialisation à un ensemble de discours. En effet, ce phénomène social pose comme défi d'articuler les deux dimensions 
des pratiques, discursives et non discursives, vérifiant ainsi la fin de la division rappellée par Roger Chartier " entre, d'un côté, le vécu, les institutions, les rapports de domination, et, d'un autre, les textes, les représentations, les constructions intellectuelles. Le réel ne pèse pas plus d'un côté que de l'autre " (Chartier, 1998, p. 143). L'analyse de la patrimonialisation requiert de savoir comment tenir ensemble ces deux réalités.

Il faut alors concevoir d'utiliser le modèle narratif greimassien, non pas comme un outil d'analyse des textes, mais comme un outil pour écrire des récits, et plus précisément pour décrire l'action représentée dans les discours des acteurs. La narratologie permet ainsi de faire apparaître l'action sociale de patrimonialisation à travers les discours recueillis en révélant leur structure organisationnelle et sans détruire leur cohérence.

Ce type d'expérience scripturaire, à travers l'écriture narrative, nous rapproche de la micro-histoire que Jacques Revel qualifie de recherche d'ordre, non pas esthétique, mais " d'ordre heuristique ", car souligne-t-il, " elle convie le lecteur à participer à la construction d'un objet de recherche ; elle l'associe à l'élaboration d'une interprétation ". Il pose clairement que " le choix d'un modèle narratif - ou, plus exactement, d'exposition - est aussi celui d'un mode de connaissance "(Revel, 1996, p. 33 et 35).

La difficulté de ce type d'approche est de ne pas se laisser entraîner vers une analyse narratologique de type littéraire mais de se situer sur un registre sociosémiotique, l'objectif étant de renvoyer au faire dont traitent et que réalisent ces discours et non à leur construction syntaxique. L'intérêt du traitement de l'opération de protection de la nature, comme opération de communication mettant en jeu de nombreux discours médiatiques, commence là où l'action déborde le texte journalistique, portant ses effets sur la mobilisation des personnes, sur la construction patrimoniale d'un territoire et de la place du parc naturel régional, sur la formation d'un système relationnel où entrent différents acteurs, porteurs d'autres actions et d'autres discours. Au-delà du texte médiatique, il faut se saisir de ces phénomènes de négociation, de rejet et d'appropriation de la valeur patrimoniale, et d'autre part du passage des actions individuelles à une logique symbolique collective.

Il faut donc se dégager de la prégnance structuraliste du modèle narratif pour le considérer non pas en termes de traitement des discours d'acteurs mais d'organisation compréhensive du texte du chercheur sous la forme narrative. Parler du " récit " du chercheur fait référence non pas à la littérature et à la subjectivité de l'auteur mais à la science du récit et au transfert de sa méthodologie d'analyse des récits vers la construction d'une forme d'organisation textuelle qui soit une manière 
de penser et de comprendre le processus de patrimonialisation.

RECONSIDÉRER LE JEU DES ACTEURS

Analyser le déplacement symbolique des objets et espaces nécessite de ne pas se focaliser sur la rationalité immédiate de l'acteur, donc de délaisser les catégories fonctionnelles des acteurs au profit des découpages culturels qu'ils font de ces entités rurales. L'acteur doit être saisi dans son inscription, à la fois dans une action interactive médiée par des objets, dans une spatialité et dans un temps mémoriel ; il est à considérer à travers toutes ses pratiques, celles discursives comprises. Il est donc nécessaire de suivre un processus relationnel complet et complexe entre dcs acteurs et entre ceux-ci et des choses en devenir patrimonial.

Le premier intérêt du schéma narratif actantiel 15 est d'offrir la possibilité de reconstituer une situation d'action élargie à différents types d'acteurs et aux objets. L'analyse se déplace d'une interaction entre acteurs à l'introduction de l'objet dans la relation. Le processus de patrimonialisation apparaît alors comme une dynamique d'ajustement, non plus unique par le jeu d'accès au pouvoir des acteurs, mais comme une dynamique plurielle d'ajustement des points de vue entre les personnes et entre celles-ci et les objets patrimoniaux. Ainsi, la question n'est pas de savoir ce qui fait patrimoine dans l'espace rural, mais qui le produit et selon quel objet.

Pour l'approche actantielle, de même que les objets se transforment pour devenir patrimoniaux, les acteurs se font dans l'action, par un jeu d'، épreuves qualifiantes "où ils acquièrent des compétences, de la légitimité. Ils deviennent les usagers de ces objets patrimoniaux, ils collaborent à la fabrication du sens par l'usage, ils deviennent les partenaires du parc naturel régional dans la requalification des espaces ruraux. Pour paraphraser Dominique Poulot, ils deviennent des " héritiers dégondés ${ }^{16}$ ", c'est-à-dire les nouveaux héritiers de ce patrimoine, en se saisissant d'objets, de biens, préalablement sortis d'un processus de transmission familiale. C'est donc le statut des acteurs qui est en jeu comme celui des objets et des espaces.

SUIVRE LE RÔLE D'UNE INSTANCE TIERCE

Comprendre le fondement de l'organisation de la relation entre l'acteur et l'objet patrimonial en question implique de considérer une position actantielle particulière, celle du destinateur. Le parc naturel régional apparaît comme cette figure du destinateur 
que Greimas nous invite à considérer, non pas à l'image d'" un souverain [...] disposant d'un savoir et d'un savoir-faire assurés ", mais de celui * qui se trouve en quête du savoir vrai et exerce, de ce fait, un faire interprétatif permanent "(Greimas, 1976, p. 25). On peut, ainsi, mettre en valeur le rôle d'une instance tierce, le parc naturel régional : redéfinir son territoire en termes de patrimoine et le donner à penser et à utiliser dans cette perspective au cours d'opérations de négociations. Cette approche incite à interroger l'impact des énoncés des différents acteurs en présence vis-à-vis de l'espace en jeu.

L'analyse narrative permet de mettre en évidence la fonction opérante de l'interprétation exercée par le destinateur dans la patrimonialisation : elle consiste à dépasser les motivations rationnelles et individuelles des acteurs au profit d'une interprétation symbolique commune. Cet actant-destinateur est selon nous la clé de lecture de l'action de patrimonialisation grâce à sa capacité à préfigurer la transformation de la relation entre l'acteur et l'objet. Pour que cette relation, de préfiguratrice, devienne effective, il est nécessaire que cet actant-destinateur enrôle des acteurs dans sa manière de concevoir le territoire, et qu'il les organise en un collectif.

\section{ARTICULER TEXTE ET CONTEXTE}

La spécificité du récit est de proposer un mode d'articulation du texte au contexte, comme l'ont soulevé des historiens comme J. Revel lorsqu'il explore trois modes d'usage du contexte au sein des écrits des historiens. "Usage rhétorique : le contexte, souvent présenté au début de l'étude, y produit un effet de réel autour de l'objet de la recherche. Usage argumentatif : le contexte présente les conditions générales au sein desquelles une réalité particulière trouve sa place, même si l'on ne va pas toujours au-delà d'une simple mise en regard des deux niveaux d'observation. Usage interprétatif, plus rarement : du contexte, on tire parfois les raisons générales qui permettraient de rendre compte de situations particulières. "(Revel, 1996, p. 25.) On voit bien comment le choix de l'accrochage des textes et du contexte dépend de la manière dont est défini ce dernier terme. Ces trois types d'articulation considèrent le contexte comme " unifié et homogène, à l'intérieur duquel et en fonction duquel les acteurs détermineraient leur choix ", poursuit J. Revel. Or, le cas de la patrimonialisation illustre la prise de position de J. Revel en faveur des " échelles d'observation " : pour aborder ce processus social, l'écriture doit se saisir d'une multiplicité d'expériences et de représentations qui contribuent à la construction des actions, et d'une pluralité de contextes plutôt que d'un contexte global. 
Le récit de l'opération de protection de la nature en Haut Forez, présenté en début d'article pour illustrer la démarche narrative, prend en fait place dans une composition narrative comprenant sept récits. Il définit l'intrigue globale qui donne sens à cette série de sept sous-intrigues que nous avons développée en systématisant la modélisation par le récit des données écrites et orales recueillies. Il s'agit de sept opérations de transformation portant sur ce même site : (I) relancer la fourme fermière d'Ambert (fabrication fromagère locale) ; (II) transférer la vie paysanne : le musée-auberge de la jasserie du CoqNoir ; (III) estiver autrement : l'estive ovine ; (IV) réhabiliter le métier de chaumier; (v) fêter la myrtille ; (VI) préserver les paysages d'Auvergne : les paysages pastoraux et naturels ; (VII) conserver le patrimoine bâti auvergnat : la place des jasseries.

La notion de programme narratif permet ainsi, d'une part, de penser et d'organiser cet empilement et, d'autre part, de raisonner en termes de contextes selon une perspective narrative. Les récits suivent le passage d'objets, d'espaces, de savoir-faire, depuis leur usage traditionnel vers des usages qui consistent à les reprendre de manière contemporaine, c'est-à-dire patrimoniale. Nous obtenons une succession de " programmes narratifs " correspondant à des "faire transformateurs ". Prenons l'exemple de la relance du produit fromager dans les monts du Forez citée dans le paragraphe précédent : ce produit existe semble-t-il depuis le Moyen Âge au sein d'une organisation agro-pastorale, sa fabrication artisanale commence dès le début du XXe siècle ; puis il passe dans l'industrie à partir des années 1960 alors que les paysans arrêtent sa fabrication traditionnelle ; tandis qu'une production fermière s'en empare à l'orée des années 1990. Si l'on reprend la terminologie greimassienne, " un état nouveau "est produit au cours de ce processus de transformation avec un renouvellement de la relation entre l'acteur et l'objet valeur. L'enjeu de cette métamorphose est la refonctionnalisation et surtout une requalification sémiotique de l'espace qui consiste à donner un nouveau système de lecture de la réalité, à doter de valeurs un espace, des gens, des séquences temporelles, des objets.

Outre la possibilité d'empilement des "récits d'initiatives " qu'elle offre, l'analyse narrative envisage leur articulation, en distinguant l'" intrigue globale "(constituant la matrice de sens patrimoniale pour le Haut Forez) et la série de sous-intrigues. L'écriture narrative, par sa capacité à composer un feuilleté de scénarios où le lecteur voit se dérouler un mouvement de transformation, chaotique et pluriel et non linéaire et unique, renouvelle la manière de penser et de comprendre la multiplicité des relations et des contextes qui se nouent dans le processus de conversion patrimoniale des espaces ruraux. 


\section{POUR UN USAGE \\ MÉTHODOLOGIQUE \\ DE LA NARRATION}

chercheur, nous racontons, c'est-à-dire quels rapports entretiennent nos récits avec les discours d'acteurs, et notre lieu socio-sémiotique objet de notre recherche avec le lieu socioéconomique objet de leurs pratiques?

Parce qu'en utilisant une écriture narrative le chercheur côtoie un univers artistique et littéraire, il se doit de mettre en jeu son inscription réelle dans le processus qu'il analyse, afin que ce qu'il en dit " ne soit ni légendaire (ou "édifiant"), ni a-topique (sans pertinence) "(de Certeau, 1975, p. 79). L'analyste de la patrimonialisation peut être considéré comme le témoin contemporain de la société qu'il étudie à un moment donné. Dans cette perspective, la production scientifique concernant ce processus fonctionne comme le témoignage d'un renouveau social et d'un écart culturel produit par l'arrivée de nouveaux acteurs, tels que les institutions " parc naturel régional17 ". Cependant, donner à la nature de l'échange entre la logique narrative et la logique historique, entre celui qui écrit l'histoire et celui qui la fait, la valeur de témoignage, n'exclut pas la particularité du rapport du texte du chercheur à la réalité. En reprenant les termes de $\mathrm{N}$. Heinich, on peut dire que restituer ce que disent et font les acteurs tout en les déplaçant de leur univers de valeurs, c'est " un autre regard, une autre façon de donner sens à leurs investissements " (1998, p. 79).

Il ne s'agit donc pas de produire reconnaissance et légitimité du travail réalisé par les acteurs mais de proposer un témoignage qui implique un point de vue scientifique : une réflexion d'ordre méthodologique sur les effets compréhensifs de la narration et une saisie des discours d'acteurs au regard de leur opérativité sociale et symbolique du point de vue de la requalification de l'espace rural. Les discours recueillis constituent une première augmentation de la réalité parce qu'ils permettent aux acteurs de donner un sens à leur action, d'installer dans l'espace public leur représentation de l'action. Le travail d'analyse consiste à prélever cette construction de sens, qui irrigue directement la réalité par les discours qui s'y insèrent, et qui sont des composants de celle-ci. Ainsi, l'organisation narrative du texte final vise à décrire l'action sociale à travers ses opérateurs discursifs.

La patrimonialisation n'étant pas simplement une affaire de langage, cette mise en forme cherche à comprendre et à rendre visible non ce qui se passe dans les discours, mais, en arrière-plan, dans la société qui les porte et les échange. Le cadre d'écriture narratif est une manière de rapporter l'expérience 
vécue de la genèse du sens patrimonial. Nous considérons que l'usage du modèle narratif constitue une seconde * augmentation " de la réalité (après celle du discours médiatique) qui possède sa spécificité : la force du récit est bien de ne pas trahir l'ordre des discours, mais de permettre de les exposer de façon à éclairer l'action qu'ils représentent, à mettre en relief les moments de perturbation où une transformation s'opère dans la relation entre acteurs et objets, à révéler la mise en place d'une organisation collective par-delà les expériences individuelles ${ }^{18}$.

Explorer ces opérations de démarcation entre le chercheur, les acteurs et le lieu de leurs pratiques n'engendre pas une fermeture sur soi, mais consiste au contraire à poser un regard sur une spécificité qui est la méthode utilisée pour apporter de la connaissance sur un objet de recherche, à savoir le processus de patrimonialisation. C'est en faisant apparaître la manière dont est fabriqué son propre texte que le chercheur se situe " au coeur du sujet19 ", c'est-à-dire à la fois au coeur de la patrimonialisation et au plus près de ce que disent et font les acteurs de ce processus.

C. $T$.

université Jean-Monnet, Saint-Ëtienne 
NOTES

1. Cet article prend appui sur notre travail de doctorat en muséologie et sciences de l'information et de la communication, La Construction patrimoniale d'un territoire. Le cas du parc naturel régional LiuradoisForez (1999).

2. En 1971, le ministre de l'Environnement Robert Poujade désigna les parcs naturels régionaux comme des * laboratoires au service d'un aménagement fin du territoire. Depuis, ce terme ne cesse de les définir. Pour notre part, ce terme nous interpelle car il transporte avec lui une interrogation sur le fonctionnement même de l'institution ainsi désignée, sur les opérations et expériences réalisées dans leurs territoires, de l'expérimentation technico-économique de l'aménagement du territoire au changement social et symbolique d'un espace rural devenu territoire " parc naturel régional ".

3. Le parc Livradois-Forez se situe sur une partie des départements du Puy-de-Dôme et de la Haute-Loire et dans la région Auvergne. Le site du Haut Forez présenté dans cet article se situe sur la limite est du parc, qui se superpose aux limites départementale et régionale entre la Loire et le Puy-de-Dôme, entre Rhône-Alpes et l'Auvergne.

4. Ce récit a été extrêmement réduit par rapport à son exposé original dans notre doctorat. Nous avons choisi de présenter un seul récit, alors que celui-ci s'insère dans un ensemble de récits, et de nombreuses citations des discours recueillis ont été supprimées. La priorité a été de conserver la structure générale du récit.

5. "Hautes Chaumes ": appellation donnée à cet espace sommital dénudé par D'Alverny en 1907 et reprise dans les années 1980 , vulgarisée par le parc Livradois-Forez notamment.

6. Ces travaux scientifiques en écologie succèdent à l'analyse développée par un chercheur en sciences sociales en 1971 (Damon, Les Jasseries des monts du Forez, thèse en sociologie rurale), et portant sur l'organisation de la vie pastorale traditionnelle. Les valeurs accordées à la partie sommitale, dite * la montagne *, sont à l'opposé de celles véhiculées par les sciences écologiques. De manière synthétique, on passe d'une conception interdépendante de * la montagne * et des versants à l'indépendance des * Hautes Chaumes ", de la recherche de l'acquisition de biens privés au patrimoine comme bien commun, de la pauvreté sociale à la richesse écologique. La mise en perspective de l'ensemble de ces travaux permet de considérer la manière évolutive et changeante de coter les objets dans le temps long de l'histoire.

7. Cette démarche est partie des entretiens avec des acteurs locaux qui nous ont toujours renvoyé aux articles de presse parus à ce sujet, d'une part parce que l'événementiel très fourni rendait son récit verbal compliqué, d'autre part parce qu'eux-mêmes avaient participé à ce débat médiatique.

8. Cette notion de * récit médiatique * est développée dans la revue Recherches en communication intitulée * Le Récit médiatique . Annik Dubied pointe le problème majeur de l'indétermination de la clôture du récit médiatique, critère qui fonde la définition du récit " classique .. "Temps de production et temps de réception se mêlent dans des récits qui s'étendent sur plusieurs années, et qu'il est délicat de délimiter strictement. • La définition du récit médiatique s'effectue selon trois niveaux de récit : le macro-récit, le méso-récit et la séquence. Le premier est défini comme * le récit-somme de tous les fragments produits à propos d'une thématique .. Le second distingue le type de médias, global tel que la télévision, particulier tel qu'un journal. En dernier lieu, 
l'analyse prend en compte la séquence narrative, * cette forme narrative élémentaire insérée dans l'unité textuelle - (Dubied, 1997, p. 161-162).

9. Le CRHP, créé en 1986, dont la devise est * conserver pour innover ", s'intéresse à différents sites régionaux sur le plan patrimonial, dont le Haut Forez.

10. L'article 19 est une aide financière de la CEE dans le cadre de l'entretien des milieux naturels par les agriculteurs. Le Haut Forez est un site sélectionné dans ce programme agri-environnemental.

11. Le Conservatoire régional de l'habitat et des paysages (CRHP) est devenu depuis 1989 le Conservatoire de l'habitat et du patrimoine naturel (CRHPN).

12. Nous reprenons les deux catégories établies par Gérard Derèze entre discours * circulants * et discours * provoqués .. " Les premiers "courent" dans la vie ordinaire, dans la vie quotidienne, dans la vie des gens ", les seconds sont " stimulés, encouragés, imposés par le chercheur qui incite les acteurs sociaux à "faire part" ou à raconter * (Derèze, 1997, p. 132).

13. D'autres matériaux discursifs ont été analysés dans le cadre de notre travail de doctorat : au centre du dispositif de gestion mis en place par les parcs naturels régionaux se trouve un texte, la charte, qui représente pour une durée de dix ans leur guide en matière d'aménagement de leur territoire. Peu contraignantes sur le plan juridique, les chartes n'en sont pas moins efficaces. D'une part, elles ont une fonction contractuelle, leur production est un moment de négociation entre les différents participants au projet territorial du parc. D'autre part, si l'on s'en tient au plus près des mots par une analyse de la structure interne et formelle du texte, on peut lire la charte non pas comme un contrat mais comme un acte (Banville et al.,
1977) d'instauration d'un nouveau territoire, d'un nouveau pouvoir et d'une nouvelle ressource environnementale et patrimoniale à gérer ensemble. En travaillant sur le cas du parc naturel régional LivradoisForez, nous avons relevé également l'exemple des conventions qui mettent en réseau différents types d'acteurs, qui authentifient et institutionnalisent le lien entre ceux-ci et un espace patrimonialisé. Là encore, ce n'est pas la dimension juridique ou non de ces documents qui est en question mais les opérations sociales et symboliques qu'ils impliquent : organisation d'une relation sociale, visibilité et institutionnalisation de l'attachement collectif à la dimension patrimoniale du territoire, apprentissage d'une représentation commune du territoire qui implique des pratiques de gestion cohérente avec celle-ci.

14. W. Sharrock et R. Watson dans leur article, * L'Unité du faire et du dire ", in Les Formes de l'action, reconsidèrent la démarche ethnométhodologique.

15. Le schéma actantiel prête attention aux relations et aux rôles qui se nouent entre les différents protagonistes, humains et non humains, au cours du récit. Il se compose de quatre positions principales : celles occupées par le sujet (le héros qui part à la quête de l'objet), le destinateur (valorise l'objet aux yeux du sujet), l'objet (à conquérir), le destinataire (reçoit le résultat de la quête et sanctionne le sujet). Au cours de sa quête de l'objet, le sujet rencontrera des opposants et / ou des adjuvants. Il faut remarquer la souplesse de ce schéma actantiel : les positions peuvent être occupées par plusieurs actants ou être vides, et surtout l'accès à une position n'est jamais définitif, d'où la possibilité de rendre compte de la circulation des actants et de leur changement de positions au cours de l'action. 
16. Dominique Poulot (1997) parle d'* héritage [...] soudainement dégondé : sorti de son cadre familier, il doit faire l'objet d'une ressaisie .

17. Les premiers * parcs naturels régionaux - remontent à 1967. Depuis, les demandes de création se sont succédé. Ils sont aujourd'hui trentesix.

18. C'est pourquoi notre intérêt ne va pas aux récits de vie ni à la trajectoire des individus, mais à la mise en réseau d'histoires.

19. Souligné par nous. Ce sont les derniers mots de l'ouvrage de Clifford Geertz (1996, p. 146).

\section{REFERENCES BIBLIOGRAPHIQUES}

Banville (E. de), Laneyrie (P.), Micoud (A.). 1977. Fonctions et enjeux des parcs naturels régionaux périurbains : l'exemple du parc du Pilat. Rapport d'étude. Saint-Étienne : CRESAL.

Bérard (L.), Marchenay (P.). 1995. - Lieux, temps et preuves. La construction sociale des produits de terroir * in Terrain, $\mathrm{n}^{\circ} 24$, mars, p. 153-164.

Certeau (M. de). 1975. L'Écriture de l'bistoire. Paris : Gallimard.

Chartier (R.). 1998. Au bord de la falaise : l'bistoire entre certitudes et inquiétude. Paris : Albin Michel.

Clifford (J.). 1996. Malaise dans la culture : l'etbnographie, la littérature et l'art au XX' siècle. Traduit de l'américain par M.-H. Sichère. Paris : Ecole nationale supérieure des beaux-arts. (Publication originale en 1988.)

Courtès (J.). 1976. Sémiotique narrative et discursive, préface de A.-J. Greimas : p. 5-25. Paris : Hachette.

Derèze (G.). 1997. "De la "vie sociale" des récits médiatiques - in Recherches en communication : " Le récit médiatique ", $n^{\circ} 7$. Louvain : université catholique de Louvain, département de Communication, p. 121-134.

Dubied (A.) 1997. * L'analyse médiatique au croisement de la narratologie et de l'ethnologie. Confrontations, délocalisations et "bricolage" - in Rechercbes en communication : "Le récit médiatique $" \mathrm{n}^{\circ} 7$. Louvain : université catholique de Louvain, département de Communication, p. 151166.

Gaudreault (A.), Jost (F.). 1990. Le Récit cinématographique. Paris : Nathan.

Geertz (C.). 1996. Ici et là-bas : l'antbropologue comme auteur. Traduit de l'anglais (USA) par D. Lemoine. Paris : Métailié. (Publication originale en 1988.) 
Greimas (A.-J.). 1976. Maupassant. La sémiotique du texte : exercices pratiques. Paris : Le Seuil.

Heinich (N.). 1998. Ce que l'art fait à la sociologie. Paris : Minuit.

Poulot (D.). 1997. Musée, nation, patrimoine, 1789-1815. Paris : Gallimard.

Revel (J.). 1996. " Présentation * et * Micro-analyse et construction du social *, p. 7-14 et 15-36 in Jeux d'écbelles : la micro-analyse à l'expérience / sous la direction de J. Revel. Paris : Le Seuil / Gallimard. Sharrock (W.) et Watson (R.). 1990. "L'unité du faire et du dire ", p. 227-253 in Les Formes de l'action. Paris : EHESS.

Tardy (C.). 1999. La Construction patrimoniale d'un territoire : le cas du parc naturel régional Livradois-Forez. Thèse de doctorat en sciences de l'information et de la communication (muséologie) : université Jean-Monnet Saint-Étienne.

Tardy (C.). 2000. * Collectionner le territoire : vers une autre collectivité ", p. 59-72 in Campagnes de tous nos désirs : patrimoines et nouveaux usages sociaux / sous la direction de $M$. Rautenberg et al. Paris : Maison des sciences de l'homme. 


\section{RÉSUMÉS}

\section{W'article commence par le récit d'une opéra-}

tion de protection de la nature dans un parc naturel régional. On voit se jouer à un premier niveau une épreuve de conversion in situ d'un espace rural en patrimoine, l'originalité résidant dans l'approche communicationnelle de ce processus de patrimonialisation. À un second niveau, cet exemple est l'occasion pour l'auteur de l'article de mettre en scène son mode d'écriture narrative comme stratégie de compréhension de ce processus. Le coeur du propos est bien là : montrer que le récit permet de traiter les discours qui circulent entre des acteurs, à propos d'objets (et plus largement d'espaces) en " devenir patrimoine ", en montrant ce qui se passe dans la société qui les porte et les échange, donc en situant la patrimonialisation comme un processus communicationnel ayant une opérativité sociale et pas seulement symbolique.

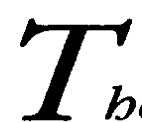

be article begins with the tale of a nature preservation project in a regional natural park. On the first level, we witness the on-site struggle to convert a rural spot into a part of the cultural beritage. The originality of this event rests in the communication aspect of this heritage-making process. On a second level, this example gives the article's author the chance to put her narrative writing style on stage as a strategy to belp us understand this process. This is the heart of the matter. The article shows that narrative allows us to deal with those discourses that circulate between acting agents concerned with objects and, more broadly speaking, with spaces that are becoming parts of the cultural heritage. This is done by showing what happens in the society that possesses and exchanges these objects and spaces. Heritage-making is thus contextualised as a communicational process that functions on a social, and not just symbolic, level.

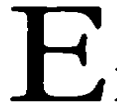

artículo empieza con el relato de una operación de protección de la naturaleza en un parque natural regional. Vemos que en un primer nivel tiene lugar una prueba de conversión in situ de un espacio rural en patrimonio, cuya originalidad reside en el enfoque comunicacional de este proceso de patrimonialización. En un segundo nivel, este ejemplo sirve de ocasión al autor del artículo para poner en escena su modo de escritura narrativa en tanto estrategia de comprensión de dicho proceso. El centro de todo radica en esto : probar que el relato permite tratar los discursos que circulan entre 
actores, a propósito de objetos, y más ampliamente de espacios, a volverse patrimonios, dando a conocer lo que pasa en la sociedad que los propulsa y los intercambia, situando, por lo tanto, la patrimonialización como un proceso comunicacional que tiene una operatividad social y no únicamente simbólica.

135

L'ENTREMISE DU RÉCIT DU CHERCHEUR 